\title{
Mandibular dentigerous cyst: Enucleation and bone reconstruction - Case report
}

\section{Duarte $F^{1}$, Ramos $C^{2}$}

\section{Affiliations:}

1. Oral Surgeon Specialist by OMD (Portuguese Dental Association)

Master of Science in Oral and Maxillofacial

Surgery at Eastman Dental Institute - University College of London

PhD student at UCL - University College of London

CEO and Clinical Director of Clitrofa - Trofa - Portugal

2. MSc student in Oral Oncology at Instituto de Ciências Biomédicas Abel Salazar - Oporto University - Portugal

\section{Corresponding author:}

Fernando Duarte

Clitrofa - Centro Médico, Dentário e Cirúrgico fduarte@clitrofa.com

\begin{abstract}
Purpose: The dentigerous cyst is the second most common odontogenic cyst in the jaws. These lesions are observed in routine examinations. The third molars and maxillary canines are the most affected teeth. Definition of marsupialization and enucleation techniques as well as consideration of important objective criteria for the treatment plan like cyst size, age, and proximity to anatomical structures, clinical importance of the tooth or teeth associated with the lesion and risk of bone fracture. This paper will present a case report treated by maneuver of decompression followed by enucleation and bone reconstruction.

Case report: A 43-year-old female patient, Caucasian, attended the Oral-Maxillofacial Surgery consultation at Clitrofa - Centro Médico, Dentário e Cirúrgico, in Trofa Portugal, to assess extraction of 3.8 and 4.8. On intraoral physical examination, a slight bulging of the cortical bone was noted in the region of the left external oblique line. Aspiration puncture was performed, and a small amount of light-yellow liquid confirmed the cystic nature of the lesion. In a second stage, osteotomy through piezoelectric surgery was performed. The cystic capsule was excised together with the extraction of tooth 3.8 included and tooth 3.7. Bone regeneration was performed. The material obtained from the cystic cavity was sent for pathological examination, confirming the diagnosis of dentigerous cyst. During observation of the patient for twelve months, no hypoesthesia or any sign of recurrence of the lesion was observed. Conclusion: The technique preserved the neurosensory function and was effective for the enucleation and reconstruction of the lesion. Despite the clinical peculiarities of each case and the chosen treatment method, the prognosis of these lesions is favourable when appropriate therapy is used.
\end{abstract}

\section{KEYWORDS}

Dentigerous cyst; odontogenic cysts; bone reconstruction 


\section{INTRODUCTION}

The dentigerous cyst is defined as a cyst that originates by the separation of the follicle from around the crown of an unerupted tooth. This is the most common type of developmental odontogenic cyst, making up about 20\% of all epitheliumlined cysts of the jaws. The dentigerous cyst encloses the crown of an unerupted tooth and is attached to the tooth at the cementoenamel junction. ${ }^{1}$

It is a benign lesion originates from the separation of the follicle around the crown of the tooth in question, forming a cavity bounded by the reduced enamel epithelium and the tooth enamel, which is filled with cystic fluid. ${ }^{2}$

As with other cysts, expansion of the dentigerous cyst is related to epithelial proliferation, release of bone-resorbing factors and an increase in cyst fluid osmolality. ${ }^{1}$

\section{Etiology and pathogenesis}

Although its etiopathogenesis is not fully known, it is believed that epithelial proliferation around a fluid-filled cavity grows continuously by osmotic pressure over an extended period, as long as the tooth does not erupt.?

Most dentigerous cysts are considered to be developmental in origin; there are some examples that appear to have an inflammatory pathogenesis. It has been suggested that, on occasion, a dentigerous cyst may develop around the crown of an unerupted permanent tooth as a result of periapical inflammation from an overlying primary tooth. Another scenario involves a partially erupted mandibular third molar that develops an inflamed cyst like lesion along the distal or buccal aspect. Although many such lesions probably are due to inflammation associated with recurrent pericoronitis, these lesions are usually diagnosed as examples of dentigerous cyst, especially because it is impossible to determine histopathologically whether the inflammatory component is primary or secondary in nature. ${ }^{1}$

\section{Histopathology}

Attachment of the cyst lining at or near the amelocemental junction suggests that dentigerous cysts arise as a result of cystic change in the remains of the enamel organ after enamel formation is complete. Division between the remnants of the internal enamel epithelium covering the enamel and the external enamel epithelium, forming the greater part of the cyst lining, can occasionally be seen at the attachment of the cyst to the neck of tooth. $^{3}$

Progressive growth of the cyst leads to dilatation of the dental follicle. Factors triggering these changes are not known. However, there is a strong association between failure of eruption of teeth and formation of dentigerous cysts which predominantly affect teeth which are particularly prone to failure of eruption, namely, maxillary canines and mandibular third molars in particular. ${ }^{3}$

The lining of dentigerous cysts typically consists of thin, sometimes bilaminar, stratified epithelium, frequently with numerous mucous cells. This epithelium may occasionally keratinise by metaplasia. The fibrous wall is similar to that of radicular cysts, but inflammatory changes are typically absent. ${ }^{3}$

\section{Radiology}

The cavity is well circumscribed, rounded and unilocular and contains the crown of a tooth displaced from its normal position. Although multilocular aspects can also occur in large lesions. ${ }^{4,5}$ Occasionally there may be pseudoloculation as a result of trabeculation or ridging of the bony wall. The slow growth of dentigerous cyst usually results in a sclerotic bony outline and a well-defined cortex. The affected tooth is often displaced a considerable distance, for instance, the third molar may be pushed to the lower border of the mandible and ultimately cause pathological mandibular fracture, ${ }^{6,7}$ Rarely, if the cyst remains unrecognized for a long period the enclosed tooth may become resorbed.

While a normal dental follicle has three to four millimeters of space between the tooth and its margin, this cyst can be suspected when this space is larger than five millimeters. ${ }^{1,8}$

These features readily distinguish a dentigerous cyst from a radicular cyst. However, a keratocyst or ameloblastoma may occasionally envelop the crown of the tooth, and either of these may create the radiographic appearance of a dentigerous cyst. The diagnose ultimately therefore depends on histological examination. ${ }^{3}$

\section{Differential diagnosis}

A differential diagnosis of pericoronal radiolucency should include odontogenic keratocyst, ameloblastoma, and other odontogenic tumors. Ameloblastic transformation of a dentigerous cyst lining should also be part of the differential diagnosis. Adenomatoid odontogenic tumor would be a further consideration with anterior pericoronal radiolucencies, and ameloblastic fibroma would be a possibility for lesions occurring in the posterior jaws of young patients. ${ }^{2}$

\section{Clinical features}

Dentigerous cysts are more than twice as common in males as females. They are uncommon in children and most often found in Caucasian individuals with ages between 20 and 50. Although this pathology may occur in any unerupted tooth, the teeth most frequently affected are the lower third molars, upper canines and lower premolars. ${ }^{1,9}$ The occurrence in primary dentition is extremely rare. ${ }^{10}$

Like other cysts, uncomplicated dentigerous cysts cause no symptoms until the swelling becomes noticeable. Alternatively, this pathology may be a chance radiographic finding or found when the cause is sought for a missing tooth. Infection of a dentigerous cyst causes the usual symptoms of pain and accelerated swelling. ${ }^{3}$ However, they can grow considerably and cause expansion of the cortical bone, facial deformation, paraesthesia and discomfort. ${ }^{11}$ They may be associated with some syndromes, when they present in multiple or bilateral forms, such as Maroteaux-Lamy and Cleidocranial Dysplasia. ${ }^{11}$

\section{Treatment options}

If the tooth is in a favorable position and space is available, it may occasionally be possible to marsupialise a dentigerous cyst to allow the tooth to erupt. Alternatively, the tooth can be transplanted to the alveolar ridge or extracted, as appropriate, and the cyst enucleated.

Marsupialization and enucleation are the classic techniques for the treatment of dentigerous cyst, and may be associated. ${ }^{4,5}$ The decompression maneuver, using a decompression device, presents itself as an option when followed by enucleation, for the treatment of the large ones. However, the criteria for choosing one of these modalities are not clearly defined, due to the lack of exhaustive studies and adequate follow-ups. As accepted criteria for diagnosis and treatment, the size of the cyst, the age of the patient, the teeth involved and the involvement of anatomical structures must be taken into account. ${ }^{1}$ The treatment modality to be chosen will depend on the clinical and radiographic characteristics in question.

Lesion aspiration should be performed in all cases, as radiographically similar lesions can be odontogenic tumors or, still, vascular lesions, and not cysts as expected, with the detection of fluid inside the lesion being a major indication of cyst. ${ }^{12,13,14}$ 
Then, the incisional biopsy must necessarily be performed to differentiate the type of cyst, as other lesions, such as the keratocystic odontogenic tumor and unicystic ameloblastoma, may have similar clinical and radiographic characteristics; however, they are more aggressive locally, requiring more extensive treatment, with sacrifice of neurovascular structures, bone and adjacent teeth. ${ }^{14}$

The prognosis of dentigerous cyst is favorable and has a low recurrence rate (3.7\%); even so, the follow-up must be tight.

Enucleation of the cyst and extraction of the associated unerupted tooth are performed in about $85 \%$ of cases and it is the treatment of choice for small lesions with safe distance to anatomical structures, such as the lower alveolar nerve., ${ }^{1,14}$ In these patients, the indication is for the unerupted tooth to be considered useless for masticatory or aesthetic function, or for lack of clinical space for its eruption. ${ }^{12}$

In dentigerous cysts from third mandibular molars, the larger the cyst, the greater the risk of nerve injury and weakening of the mandibular angle caused by the surgery. Therefore, in these cases, the most appropriate therapeutic modality will be the decompression maneuver followed by the enucleation technique, after reducing the size of the lesion. . $^{15,16,17}$

\section{Bone reconstruction}

The two-stage treatment is time-consuming, uncomfortable for patients and requires frequent check-ups. One-stage cystectomy of large cysts with water-tight closure of the postoperative bone cavity predisposes to complications. Moreover, the weakened bone structure is prone to fractures in the postoperative period. This is why there is particular interest to fill the bone cavities with autografts or bone graft substitutes.

\section{Clinical case}

A 43-year-old female patient, Caucasian, attended the OralMaxillofacial Surgery consultation at Clitrofa - Centro Médico, Dentário e Cirúrgico, in Trofa - Portugal, to assess extraction of 3.8 and 4.8. She was asymptomatic, without paresthesia, hypoesthesia or other complaints.
After anamnesis, there was no an allergy or use of medications. On extraoral clinical examination, an aspect of normality was observed. On intraoral physical examination, a slight bulging of the cortical bone was noted in the region of the left external oblique line, adjacent to tooth 3.7, with no chromatic alteration in the oral mucosa.

The orthopantomography showed a unilocular, well-defined, homogeneous radiotransparent image that surrounded the dental crown of the included 3.8 tooth, extending to the 3.5 tooth region (Figure $1 \mathrm{~A}$ ). In computed tomography in coronal, sagittal and axial sections, it can be seen that the lesion was in close contact with the mandibular canal and there was cortical bulging (Figure 1B and Figure 1C).

As initial hypotheses for diagnosis, the possibilities of keratocystic odontogenic tumor, unicystic ameloblastoma, adenomatoid odontogenic tumor and dentigerous cyst were considered.

Aspiration puncture was performed under local anesthesia, with a small amount of light-yellow liquid, confirming the cystic nature of the lesion and working as decompression maneuver to reduce the lesion size. Endodontic treatment of tooth 3.6 was performed prior to surgical intervention.

The patient was operated under general anesthesia, with nasal intubation. An intraoral incision was made in the left retro molar region that extended to the canine region, where a discharge incision was made. The osteotomy of access to the cystic cavity was performed by piezoelectric surgery, based on the threedimensional control of ultrasonic microvibrations, a micrometric and selective cut is allowed, with good visibility (cavitation effect) that result in minimal damage to soft tissue and nervous structures (Figure 2A). ${ }^{6}$ The equipment used was the VarioSurg $3^{\circ}$ $\left(\mathrm{NSK}^{\circ}\right)$.

The cystic capsule was excised together with the extraction of tooth 3.8 included and tooth 3.7 (Figure 2B). The remaining cavity was cleaned with saline solution, maintaining the lower border of the mandible intact (Figure 2C).

Bone regeneration was performed with Cerasorb $\mathrm{M}^{\circ}$ (Curasan ${ }^{\circ}$ ) in granules and foam combined with fibrin and Osgide resorbable membrane (Curasan ${ }^{\circ}$ ). Cerasorb $M^{\circ}$ is a resorbable and a pure

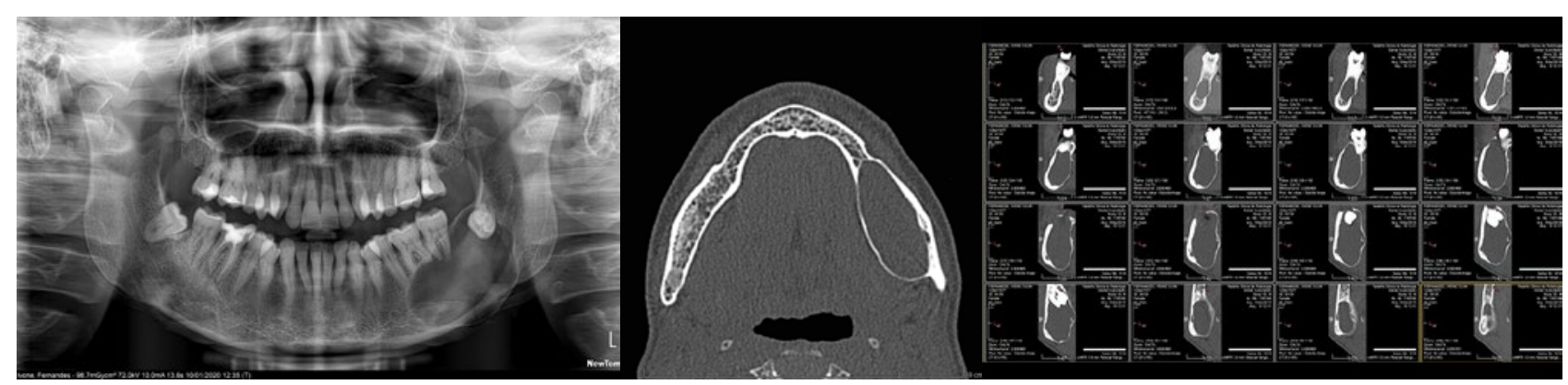

Figure 1. (A) Initial orthopantomography, (B) Initial computed tomography sagittal section, (C) Initial computed tomography cross section

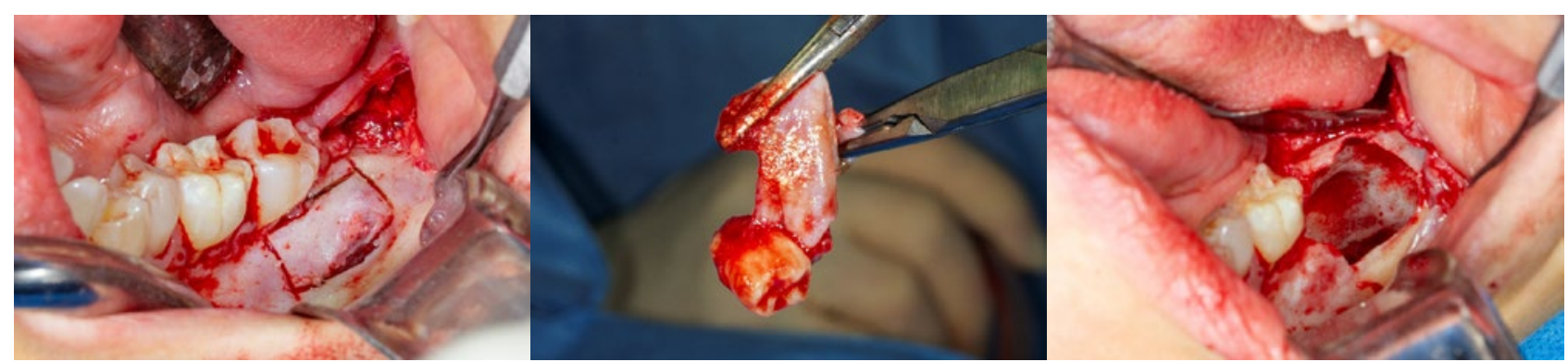

Figure 2. (A) Osteotomy with piezoelectric surgery, (B) Cystic capsule with impacted 3.8, (C) Intraoperative image of the cystic cavity 
phase tricalcium beta-phosphate ceramics for implantation filling, binding, and reconstruction of bone defects as well as for bone fusion in the entire skeletal system. The granules have a polygonal shape and, due to the open intercommunicating multiporosity composed of micro, meso and macropores (about 65\%), the radiopacity is lower and absorption is effected faster. Over the months, in contact with the vital bone, the material is resorbed by the body and simultaneously replaced by autologous bone tissue. As a synthetic and bioactive ceramic material, Cerasorb $\mathrm{M}^{\circ}$ has no local or systemic toxicity and no risk of allergic reaction. Cerasorb $\mathrm{M}^{\circledR}$ is radiopaque and can be used in granules, paste and foam. ${ }^{18}$

The use of autologous leukoplakelet fibrin (PRF) in the graft process allows to enjoy its characteristics, namely in the modeling of the inflammatory response, immune response and tissue repair, tissue reorganization and angiogenesis (Figure $3 \mathrm{~A}) .{ }^{19}$

The association of PRF with mineral biomaterials (I-PRF) facilitates handling, application and allows immediate adhesion to the receiving bed (Figure $3 \mathrm{~B}$ and Figure $3 \mathrm{C}$ ). ${ }^{19}$

The inferior layer of bone reconstruction was made with Cerasorb $M^{\circ}$ foam soaked with PRF in an attempt to protect the inferior alveolar nerve integrity and strengthen the lower border of the mandible.

The superior reconstruction layer was done with Cerasorb $\mathrm{M}^{\circ}$ granules combined with PRF, in what is described as sticky bone. Sticky bone provides stabilization of bone graft in the defect, is easy to manipulate, and therefore, accelerates tissue healing and minimizes bone loss during healing period (Figure 4A).

Osgide ${ }^{\circ}$ (Curasan ${ }^{\circ}$ ) resorbable membrane was used to cover the bone reconstruction. Osgide is a bioresorbable barrier membrane for use in guided tissue regeneration (GTR) and guided bone regeneration (GBR). The membrane creates a protected environment for bone regeneration in the defect area and supports osteoneogenesis by presenting a barrier to the infiltration (migration) of soft tissue and promotes the growth of osteogenic cells in the bone defect (Figure 4B). Suture was performed with simple stitches using non-resorbable thread (Silk 4.0) (Figure 4C).

The patient underwent systemic antibiotic, analgesic and antiinflammatory therapy for 8 days.

In postoperative care, the patient was instructed to maintain strict oral hygiene. The material obtained from the cystic cavity was sent for pathological examination, and the result reports: islands of odontogenic epithelium remains scattered in the fibrous connective tissue capsule composed of three layers of flattened, non-keratinized cells. These findings confirmed the diagnosis of dentigerous cyst.

The patient is in the postoperative period of twelve months, without hypoesthesia or any sign of recurrence of the lesion. In postoperative orthopantomography (Figure 5A) and computed tomography, there is evidence of bone neoformation in the area previously occupied by the lesion (Figure 5B and Figure 5C).

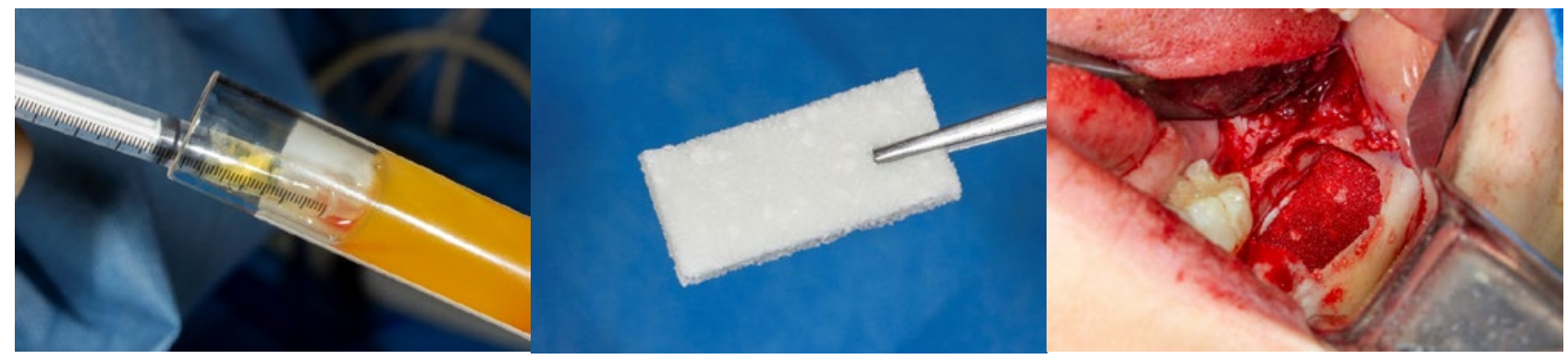

Figure 3. (A) Leukoplakelet fibrin aspect, (B) Cerasorb $\mathrm{M}^{\circ}$ foam used to protect the inferior alveolar nerve, (C) Intraoperative image of inferior layer of bone reconstruction

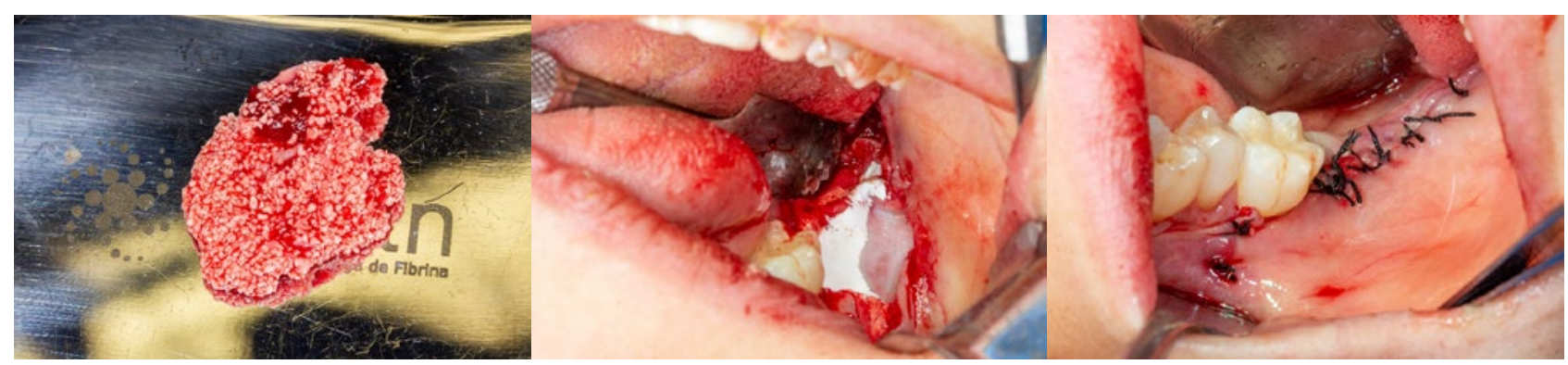

Figure 4. (A) Sticky bone for cyst cavity reconstruction, (B) Osgide" resorbable membrane covering all bone reconstruction, (C) Intraoperative a after suture

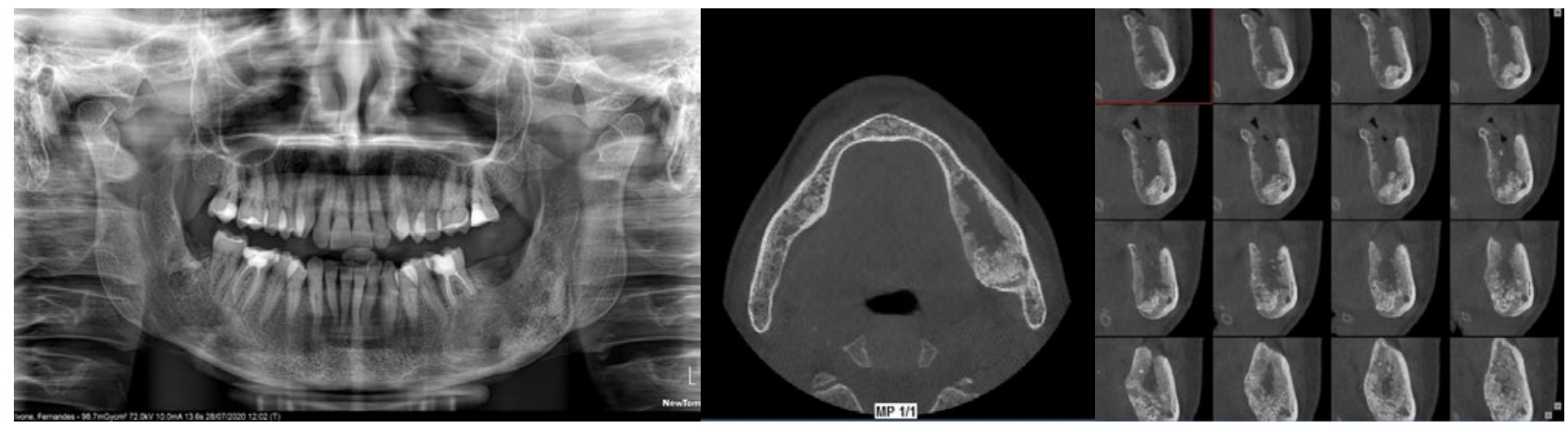

Figure 5. - (A) Final orthopantomography, (B) Final computed tomography sagittal section, (C) Final computed tomography cross section 


\section{DISCUSSION}

Among the possible treatment techniques for dentigerous cyst, the most suitable for each clinical situation should be evaluated, considering all scenarios for each option. ${ }^{5}$ In the present clinical case, if the treatment was decided considering only the size of the lesion, enucleation would have been the most appropriate choice associated with the extraction of the impacted 3.8. Due to the proximity of the inferior alveolar nerve, the decompression technique, followed by cyst enucleation and extraction of the tooth in question was the most reasonable option. This allowed bone regeneration of the cystic cavity, as well as maintain nerve integrity.

Enucleation is the technique of choice for cysts of smaller dimensions and when there is no involvement of important anatomical structures, since it provides the possibility of a histological study of the lesion. ${ }^{1,14}$

The marsupialization technique and the decompression maneuver with the use of a device should be reserved for the clinical cases of extensive dimensions, involving important anatomical structures and increased risk of fractures, as they promote the reduction of intracystic pressure, with a consequent reduction in the size of the lesion. $4,5,15$ Taking into account this objective of the decompression maneuver, it was decided in this clinical case to maintain the lesion-oral cavity communication created during the aspiration puncture, which, although reduced, was sufficient to regress the dimensions of the lesion. Both the marsupialization technique and the decompression maneuver are therapeutic modalities also indicated during the period when the bone repair capacity is high, and the eruptive power of the teeth is present.

Sticky bone is biologically solidified bone graft which is entrapped in fibrin network. The sticky bone graft granules strongly interconnected each other by fibrin network. Sticky bone has numerous advantages: 1) it is moldable, so well adapted over various shape of bony defect; 2) Micro and macro movement of grafted bone is prevented. So the volume of augmentation is maintained during healing period, therefore the need of block bone and titanium mesh is minimized; 3) Fibrin network entraps platelets and leukocytes to release growth factors, so bone regeneration and soft tissue is accelerated; 4) No biochemical additives are needed to make sticky bone; 5) Fibrin interconnection minimizes soft tissue ingrowth into the sticky bone graft. ${ }^{19}$

\section{CONCLUSION}

The dentigerous cyst is a frequent lesion, which despite being a less aggressive pathology and without clinical symptoms has the potential to reach large proportions, causing significant dental movements.

The decision about the treatment of choice must be based on objective criteria, such as the age of the patient, the size of the lesion, involvement of relevant anatomical structures, the clinical importance of the tooth or teeth associated with the lesion and risk of bone fracture.

It is essential to perform a histopathological examination for the differential diagnosis, ruling out other types of lesions with similar clinical and radiographic characteristics, in addition to the annual postoperative radiographic monitoring.

\section{CONFLICT OF INTEREST}

The authors declared that there is no conflict of interest.

\section{REFERENCES}

1. Neville BW, Damm DD, Allen CM, Bouquot JE. Oral \& Maxillofacial Pathology. 2nd edition. United States of America: W. B. Saunders Company; 2002

2. Jena AK, Duggal R, Roychoudary A, Prakash H. Orthodontic assisted tooth eruption in a dentigerous cyst: a case report. J Clin Pediatr Dent. 2004 Fall;29(1):33-5.

3. Cawson RA, Odell EW. Cawson's Essentials of Oral Pathology and oral Medicine. 7th edition. London: Churchill Livingstone; 2002.

4. Goaz PW, Stuart CW. Cysts of the jaws. Oral radiology, principles and interpretation. 3rd ed. St Louis: Mosby; 1994.

5. Zhang LL, Yang R, Zhang L, Li W, MacDonald JD, Poh CF. Dentigerous cyst: a retrospective clinicopathological analysis of 2082 dentigerous cysts in British Columbia, Canada. Int J Oral Maxillofac Surg. 2010 Sep;39(9):878-82.

6. Vercellotti T, De Paoli S, Nevins M. The piezoelectric bony window osteotomy and sinus membrane elevation: introduction of a new technique for simplification of the sinus augmentation procedure. Int J Periodontics Restorative Dent. 2001 Dec;21(6):561-567.

7. Lustig JP, Schwartz-Arad D, Shapira A. Odontogenic cysts related to pulpotomized deciduous molars - clinical features and treatment outcome. Oral Surg Oral Med Oral Pathol Oral Radiol Endod. 1999 Apr;87(4):499-503.

8. Goaz PW, Stuart CW. Cysts of the jaws. Oral radiology, principles and interpretation. 3rd ed. St Louis: Mosby; 1994.

9. Takagi S, Koyama S. Guided eruption of an impacted second premolar associated with a dentigerous cyst in the maxillary sinus of a 6-year-old child. J Oral Maxillofac Surg. 1998 Feb;56(2):237-39

10. Boyczuk MP, Berger JR. Identifying a deciduous dentigerous cyst. J Am Dent Assoc. 1995 May;126(5):643-44.

11. Ko KSC, Dover DG, Jordan RCK. Bilateral dentigerous cysts - report of an unusual case and review of the literature. J Can Dent Assoc. 1999 Jan;65(1):49-51.

12. Ertas Ü, Yavuz S. Interesting eruption of 4 teeth associated with a large dentigerous cyst in mandible by only marsupialization. J Oral Maxillofac Surg. 2003 Jun;61(6):728-30.

13. Benn A, Altini M. Dentigerous cysts of inflammatory origin - a clinicopathologic study. Oral Surg Oral Med Oral Pathol Oral Radiol Endod. 1996 Feb;81(2):203-9.

14. Murakami A, Kawabata K, Suzuki A, Murakami S, Ooshima T. Eruption of an impacted second premolar after marsupialization of a large dentigerous cyst: case report. Pediatr Dent. 1995 Sep-Oct;17(5):372-4

15. Monteyecchi M, Checchi V, Bonetti GA. Management of a deeply impacted mandibular third molar and associated large dentigerous cyst to avoid nerve injury and improve periodontal healing: case report. J Can Dent Assoc. 2012 Mar;78(2):59-62.

16. Hyomoto M, Kawakami M, Inoue M, Kirota T. Clinical conditions for eruptions of maxillary canines and mandibular premolars associated with dentigerous cysts. Am J Orthod Dentofacial Orthop. 2003 Nov;124(5):515-20.

17. Ziccardi VB, Eggleston TI, Schneider RE. Using fenestration technique to treat a large dentigerous cyst. J Am Dent Assoc. 1997 Feb;128(2):201-5.

18. Harel N, Moses O, Palti A, Ormianer Z. Long-term results of implants immediately placed into extraction sockets grafted with B-TCP: A retrospective study. J Oral Maxillofac Surg. 2013, 71(2): e63-e68.

19. Duarte deAlmeida FL, de Oliveira LA. The “Ring-Shaped”Autologous Fibrin Matrix:A Technical Note. Int J Growth Factors and Stem Cells in Dent. 2019 Jan-Apr;2(1):18-21. 